ROM2F/2009/21

UPR-1214-T

\title{
Branes and instantons at angles and the F-theory lift of $O(1)$ instantons ${ }^{1}$
}

\author{
M. Cvetič* ${ }^{*}$ I. García-Etxebarria* and R. Richter ${ }^{\dagger}$ \\ *Department of Physics and Astronomy, University of Pennsylvania, \\ Philadelphia, PA 19104-6396, USA \\ †Dipartimento di Fisica, Università di Roma "Tor Vergata" \\ I.N.F.N. - Sezione di Roma "Tor Vergata" \\ Via della Ricerca Scientifica, 1 - 00133 Roma, ITALY
}

\begin{abstract}
We discuss the physics of D-branes and D-brane instantons intersecting at angles, focusing on the (non)generation of a superpotential in the worldvolume theory of the branes. This is a short review of the results in arXiv:0905.1694, where we further emphasize both the macroscopic and microscopic structure of the manifestly supersymmetric instanton action. We also comment on the lift of $O(1)$ instantons to F-theory.
\end{abstract}

Keywords: Nonperturbative techniques, D-branes, String and brane phenomenology

PACS: $11.25 . \mathrm{Sq}, 11.25 . \mathrm{Uv}, 11.25 \mathrm{Wx}$

\section{INTRODUCTION}

In the last few years there has been growing interest in non-perturbative effects coming from Euclidean D-brane instantons [1], mainly due to the realization [2, 3, 4] that they could be used for solving some longstanding difficulties in string model building coming from perturbative symmetries. We refer the reader to [5] for a review on many of the recent developments in this topic.

Since [5] appeared there has been some progress in the topic. In these notes we will review some of the results in [6], putting them in a somewhat broader perspective. In particular, we will discuss how supersymmetry determines the result almost uniquely, in a manner compatible with both our global macroscopic arguments and our microscopic CFT computations. We will also describe some results about the lift to F-theory of $O(1)$ instantons.

We will focus on instantons contribution to the superpotential. In order for an instanton to contribute to the superpotential, it must have exactly two unsaturated fermionic zero modes, corresponding to the two $\theta^{\alpha} \mathscr{N}=1$ superspace fermionic coordinates $(\alpha \in\{1,2\})$ over which we integrate the superpotential.

\footnotetext{
${ }^{1}$ Based in part on talks given at Supersymmetry'09 (M.C.), Strings'09 (M.C.), CERN (I.G.-E.) and Simons Workshop in Mathematics and Physics, Stony Brook (M.C.).
} 
A generic rigid D-brane instanton in a type II background preserving 8 supersymmetries will have at least 4 fermionic zero modes living on its worldvolume, coming from the broken background supersymmetries. We will call these modes $\theta^{\alpha}$ and $\bar{\tau}_{\dot{\alpha}}$. These zero modes are the Goldstinos of the supersymmetries broken by the D-brane (which is a 1/2 BPS state). Only those instantons for which the $\bar{\tau}$ modes are projected out or lifted in going from $\mathscr{N}=2$ to $\mathscr{N}=1$ can contribute to the superpotential.

There are various known ways in which the $\bar{\tau}$ modes can get lifted in a realistic $\mathscr{N}=1$ compactification. The simplest class of instantons correspond to the lift to string theory of gauge theory instantons. They are given by D-brane instantons wrapping the same cycle as a background gauge brane 2 [7, 8, 9, 10, 11, 12, 13]. The case with a single space-filling brane (the so-called $U(1)$ instanton) is slightly special, since it does not admit a gauge theory interpretation, but the lifting of $\bar{\tau}$ modes still takes place [14, 15, 16, 17, 18].

Another class of contributing instantons are those which have a worldvolume $O(1)$ gauge symmetry, due to being invariant under an appropriate background orientifold. The orientifold will project out the $\bar{\tau}$ modes [19, 20, 12, 13].

In a realistic compactification we will also need to introduce background fluxes. These fluxes have a potentially dramatic effect on the instanton, and in some cases they can also lift the $\bar{\tau}$ modes [21, 22, 23, 24, 25, 26, 27, 28, 29, 30, 31, 32, 33, 34, 35]. This is generically hard to do without breaking supersymmetry [31, 32, 33, 34, 35], for reasons best understood from the effective field theory analysis [32, 35]. In [6] we introduced a configuration, connected to $\mathscr{N}=4$ instantons in their Coulomb branch, in which flux lifts $\bar{\tau}$ modes, and which can be studied using open string techniques. We will review it below.

So far these are the only known configurations in which a D-brane instanton can contribute to the low-energy superpotential. It is an interesting open problem to determine whether these are all the possibilities or other configurations exist. Particularly interesting is the case where background branes intersect the instantons at angles, since this is a very generic occurrence in string compactifications. If the brane intersecting the instanton could also lift the $\bar{\tau}$ modes of the instanton this would allow for great flexibility in model building. As an example, related configurations where explored in [36, 37] as a possible source of interesting non-perturbative dynamics in local F-theory models.

In order to determine the fate of this class of systems, in [6] we performed a careful analysis of a IIA system composed of a D6 brane and a rigid D2-brane instanton intersecting at angles in the internal space in the absence of orientifolds or fluxes, which we summarize here. Our main result is that in this case the $\bar{\tau}$ modes are not lifted, and thus no superpotential is generated by the D-brane instantons 3 Again, this negative result has important implications for string theory model building, since generic configura-

\footnotetext{
${ }^{2}$ More precisely, they must wrap the same cycle and have identical worldvolume bundle. If the worldvolume bundle is different the instanton is not an ordinary field theory gauge instanton, and as we will show below it does not contribute to the superpotential.

${ }^{3}$ Of course, some other mechanism such as fluxes or orientifolds can lift the zero modes of the instanton in our setup, allowing it to contribute to the superpotential. What we show here is that such a lifting cannot come from the background brane intersecting the instanton at an angle.
} 
tions cannot generate certain desired interactions, such as Polonyi-type couplings.

The main technical tool we will use in our analysis is a microscopic CFT calculation, although we will also discuss some general effective field theory arguments forbidding the generation of the superpotential. This is the main reason why we have chosen to work in type IIA: close to the point(s) where the instanton and the brane intersect one has an explicit conformal field theory (CFT) description of the system, and the fact that $\bar{\tau}$ modes are not lifted can be shown in detail.

We will be able to extract some lessons from the CFT analysis that we expect to be of general applicability. The most important one concerns the supersymmetry of the system. As we will see, obtaining a supersymmetric instanton action is a very constraining requirement, which our CFT results satisfy in a non-trivial manner. Related to this, we will find that in the intersecting case the spectrum of states is markedly different from the one we obtain in the gauge theory case, so one should exercise care in generalizing expressions obtained from gauge theory instantons.

The type IIA intersecting brane picture is related by duality to many interesting string backgrounds, so our result has a wide range of applicability. We refer the reader to [6] for details and references on some known dual configurations where our results have important consequences.

\section{CFT ANALYSIS OF INSTANTONS AT ANGLES}

\section{General structure of the instanton action}

Before going into the detailed CFT analysis, let us highlight some general features of our analysis.

The first important result we obtain from the CFT analysis concerns the spectrum of massive modes, shown in table 1. We will find that in addition to the modes that appear in the gauge instanton limit, there exist some additional modes (which we will denote below by primes) that only appear in the intersecting case, and have no analog in the gauge instanton case. They are required in order to give a mass to the modes that become massless in the gauge instanton limit. One essential feature is that $\mu$ modes pair only with $\bar{\mu}^{\prime}$ modes, not with $\bar{\mu}$ modes, as it would be naively expected. This is straightforward to see from our calculations below, since the mass of $\mu$ becomes different from the mass of $\bar{\mu}$ under small non-supersymmetric rotations of the system.

Furthermore, we will find that the supersymmetry transformations shown in equation (6) play a crucial role in constraining the action. Looking at these supersymmetry transformations it is easy to see that the mass couplings for the $\mu$ modes have to be of the form $\mu \bar{\mu}^{\prime}$, agreeing with the general result based on the expressions for the masses.

More important than the mass couplings, though, are the cubic couplings in the effective action, shown in equation (8). Whether or not we saturate the $\bar{\tau}$ modes will depend on the form of these couplings. The couplings given in (8) are obtained using CFT, but agree beautifully with the requirement of supersymmetry. It is easy to see that any other possible coupling one might consider $\left(\bar{\tau}^{\dot{\alpha}} \bar{\omega}_{\dot{\alpha}} \mu^{\prime}\right.$ is one example one might be tempted to write) will break supersymmetry. More generally, supersymmetry and the form of the coupling to the auxiliary field $\vec{D}$ impose that the cubic coupling must be of 
the schematic form:

$$
\bar{\tau}_{\dot{\alpha}} \delta_{\xi_{\dot{\alpha}}}(\bar{\omega} \omega)
$$

In particular, only fields that appear after supersymmetry variations of $\omega$ or $\bar{\omega}$ can couple to $\bar{\tau}$. In particular this excludes $\bar{\mu}^{\prime}$, the mass partner of $\mu$.

This last property is extremely important and robust, and we believe that ultimately it is this fact that underlies the null result that we find (at least microscopically, see below for the macroscopic effective field theory justification). A similar supersymmetry analysis is also possible in related systems that do not admit a direct CFT description, as long as we have some way of determining the action of supersymmetry on the spectrum of instanton modes, 4

\section{Detailed analysis}

Let us proceed to the microscopic analysis. In type IIA the relevant instantons are Euclidean D2 branes wrapping a rigid three-cycle $\Sigma$ away from the orientifold plane in the internal manifold. Generically, such an isolated $U(1)$ instanton exhibits four bosonic zero modes $x^{\mu}$ corresponding to the breakdown of four-dimensional Poincaré invariance and various Goldstino modes $\theta_{A}^{\alpha}$ and $\bar{\tau}_{A}^{\dot{\alpha}}$ associated with the background supersymmetries broken by the instanton. The $A$ subindex comes from flat space, and runs from 1 to 4. As explained above, we will identify one of the flat space supersymmetries as the restriction to the intersection of the curved space supersymmetry. This naturally singles out one of the $A$, which we will take in our analysis to be $A=0$. We will return to this point below.

The space-time filling D6-brane wraps a cycle $\Sigma^{\prime}$ distinct from $\Sigma$, and it will intersect $\Sigma$ at isolated points in the internal space. Thus in addition to the neutral instanton modes there are also charged modes which correspond to strings stretching between the E2instanton and the D6-brane.

The different massive and massless charged instanton modes appear as excitations of the NS- and R-vacuum. Schematically they take the form

$$
\sum_{k \varepsilon \mathbf{Z}}\left(\alpha_{k-\theta_{I}}^{I}\right)^{N_{k}^{I}}\left(\psi_{k+\frac{1}{2}-\theta_{I}}^{I}\right)^{M_{k}^{I}}|0\rangle_{N S} \quad \sum_{k \varepsilon \mathbf{Z}}\left(\alpha_{k-\theta_{I}}^{I}\right)^{N_{k}^{I}}\left(\psi_{k-\theta_{I}}^{I}\right)^{M_{k}^{I}}|0\rangle_{R}
$$

where $\theta_{I}$ is the intersection angle between the D-brane and the instanton in the $I$-th dimension (we take $\theta_{I}$ to be defined in the range $[-1,1)$ ) and $\alpha_{k}^{I}$ and $\psi_{k}^{I}$ denote the bosonic and fermionic creation operators. Note that the fermionic creation operator $\psi_{k}^{I}$ has Fermi statistics, and thus $M_{k}^{I}$ can only take the values 0 or 1 , while $N_{k}^{I}$ can be any non-negative integer.

\footnotetext{
${ }^{4}$ More precisely, for all compactifications with $(0,2)$ worldsheet supersymmetry the $U(1)$ worldsheet charge is inherently related to the space-time supercharge [38, 39]. Using this fact, one can demonstrate that the $U(1)$ charge ensures a unique target space supersymmetric structure of the instanton action, given by the structure that we obtain in equations (7) and (8).
} 
The mass of these states is given by:

$$
\begin{array}{rlrl} 
& \text { NS-sector: } & & M^{2} \sim \varepsilon_{0}^{N S}+\sum_{k}\left(N_{k}^{I}\left(k-\theta_{I}\right)+M_{k}^{I}\left(k+\frac{1}{2}-\theta_{I}\right)\right) \\
\text { R-sector: } & M^{2} \sim \varepsilon_{0}^{R}+\sum_{k}\left(N_{k}^{I}\left(k-\theta_{I}\right)+M_{k}^{I}\left(k-\theta_{I}\right)\right),
\end{array}
$$

where $\varepsilon_{0}^{N S, R}$ denotes the zero point energy in the NS or R sectors, and crucially depends on the intersection angles of the D6-brane and the E2-instanton. Note that due to the Dirichlet-Neumann boundary conditions in spacetime the zero point energy for the NS sector is shifted by $1 / 2$ compared to the D6-D6 brane system. This implies that there are no tachyonic modes between the D-brane and the instanton and for non-trivial angles the bosonic modes are always massive. The absence of tachyons in this setup rules out the possibility of recombination of the instanton and D-brane. For the R-sector the Dirichlet-Neumann boundary conditions in spacetime imply that only states whose $U(1)$-wordsheet charge is $Q_{W S}=-\frac{1}{2} \bmod 2$ are allowed, thus only chiral modes survive the GSO-projection [2, 3, 4, 40]. The latter is related to the fact that the total fermion number in the R-sector is constrained due to the GSO-projection.

For concreteness we assume the intersection pattern

$$
\theta_{1}>0 \quad \theta_{2}>0 \quad \theta_{3}<0
$$

and look at all states whose mass squared is at most of linear order in the intersecting angles $\theta_{I}$. In table 1 we present all states and their corresponding mass. When the Dinstanton D-brane system preserves the supercharge $\bar{Q}_{\dot{\alpha}}^{A}$ the charged instanton modes are related to each other in the following way [6]

$$
\delta_{\bar{\xi}^{A}} \mu_{A}^{\prime}=i \bar{\xi}_{\dot{\alpha}}^{A} \omega^{\dot{\alpha}} \quad \delta_{\bar{\xi}^{A}} \omega_{\dot{\alpha}}=i \bar{\xi}_{\dot{\alpha}}^{A} \mu_{A} \quad \delta_{\bar{\xi}^{A}} \bar{\mu}_{A}^{\prime}=i \bar{\xi}_{\dot{\alpha}}^{A} \bar{\omega}^{\dot{\alpha}} \quad \delta_{\bar{\xi}^{A}} \bar{\omega}_{\dot{\alpha}}=i \bar{\xi}_{\dot{\alpha}}^{A} \bar{\mu}_{A} .
$$

If $\mu_{A}$ or $\bar{\mu}_{A}$ is massless we expect the absence of $\bar{\mu}_{A}^{\prime}$. This is analogous to the D6-D6 brane configuration, where we have in the massive case a hypermultiplet (consisting of two chiral multiplets) while in the massless case we only have a single chiral multiplet. In case the D-instanton and D-brane wrap the same cycle all supercharges $\bar{Q}_{\dot{\alpha}}^{A}$ are preserved and all $\mu_{A}$ and $\bar{\mu}_{A}$ are massless. Then the SUSY transformations reduce to the second and fourth equations of (6) which are exactly the usual SUSY transformations appearing in the ADHM case [41, 9]5.

To each state corresponds a vertex operator and one can explicitly show [6] the existence of the mass terms

$$
S_{E 2}^{\text {mass }}=\mu_{A} \bar{\mu}_{A}^{\prime}+\omega \bar{\omega}+\mu_{A}^{\prime} \bar{\mu}_{A}
$$

\footnotetext{
${ }^{5}$ Note that for the gauge instanton setup, which corresponds to all three intersection angles $\theta_{I}$ being 0 , the vacuum is defined differently. In that case the $\mu_{A}^{\prime}$ and $\bar{\mu}_{A}^{\prime}$ modes are projected out and do not appear in the instanton mode spectrum.
} 
TABLE 1. Instanton D-brane spectrum. We have paired modes by mass. Notice that, despite what the notation might suggest, $\mu$ modes can only pair up with $\bar{\mu}^{\prime}$ modes, and $\mu^{\prime}$ modes can only pair up with $\bar{\mu}$ modes. Notice also the chiral spectrum at the massless level, encoded in the fact that $\mu_{3}$ has no mass partner.

\begin{tabular}{ccccc}
\hline E2-a & CFT state & a-E2 & CFT state & mass $^{2}$ \\
\hline$\mu_{0}$ & $\psi_{-\theta_{1}} \psi_{-\theta_{2}}|0\rangle_{E 2 a}^{R}$ & $\bar{\mu}_{0}^{\prime}$ & $\frac{1}{2}\left(\psi_{-\theta_{1}} \alpha_{-\theta_{2}}+\psi_{-\theta_{2}} \alpha_{-\theta_{1}}\right)|0\rangle_{a E 2}^{R}$ & $\theta_{1}+\theta_{2}$ \\
\hline$\mu_{1}$ & $\psi_{-\theta_{1}} \psi_{\theta_{3}}|0\rangle_{E 2 a}^{R}$ & $\bar{\mu}_{1}^{\prime}$ & $\psi_{\theta_{3}} \alpha_{-\theta_{1}}|0\rangle_{a E 2}^{R}$ & $\theta_{1}-\theta_{3}$ \\
\hline$\mu_{2}$ & $\psi_{-\theta_{2}} \psi_{\theta_{3}}|0\rangle_{E 2 a}^{R}$ & $\bar{\mu}_{2}^{\prime}$ & $\psi_{\theta_{3}} \alpha_{-\theta_{2}}|0\rangle_{a E 2}^{R}$ & $\theta_{2}-\theta_{3}$ \\
\hline$\mu_{3}$ & $|0\rangle_{E 2 a}^{R}$ & & None & 0 \\
\hline$\mu_{0}^{\prime}$ & $\alpha_{\theta_{3}}|0\rangle_{E 2 a}^{R}$ & $\bar{\mu}_{0}$ & $\psi_{\theta_{3}}|0\rangle_{a E 2}^{R}$ & $-\theta_{3}$ \\
\hline$\mu_{1}^{\prime}$ & $\alpha_{-\theta_{2}}|0\rangle_{E 2 a}^{R}$ & $\bar{\mu}_{1}$ & $\psi_{-\theta_{2}}|0\rangle_{a E 2}^{R}$ & $\theta_{2}$ \\
\hline$\mu_{2}^{\prime}$ & $\alpha_{-\theta_{1}}|0\rangle_{E 2 a}^{R}$ & $\bar{\mu}_{2}$ & $\psi_{-\theta_{1}}|0\rangle_{a E 2}^{R}$ & $\theta_{1}$ \\
\hline$\mu_{3}^{\prime}$ & $\frac{1}{3} \varepsilon_{i j k}^{2} \psi_{-\theta_{i}} \psi_{-\theta_{j}} \alpha_{-\theta_{k}}|0\rangle_{E 2 a}^{R}$ & $\bar{\mu}_{3}$ & $\psi_{-\theta_{1}} \psi_{-\theta_{2}} \psi_{\theta_{3}}|0\rangle_{a E 2}^{R}$ & $\theta_{1}+\theta_{2}-\theta_{3}$ \\
\hline$\omega^{\dot{\alpha}}$ & $|0\rangle_{E 2 a}^{N S}$ & $\bar{\omega}^{\dot{\alpha}}$ & $|0\rangle_{E 2 a}^{N S}$ & $\frac{1}{2}\left(\theta_{1}+\theta_{2}-\theta_{3}\right)$ \\
\hline & & & &
\end{tabular}

In order to simplify the expression we have omitted the exact mass coefficient, it agrees with the result displayed in table 1, Note in particular that in the case where all SUSY is broken by the E2-D6 system the masses of $\mu_{A}$ and $\bar{\mu}_{A}$ are different, and thus a mass term $\mu_{A} \bar{\mu}^{A}$ is never allowed. Moreover, in case some supercharge $\bar{Q}_{\dot{\alpha}}^{A}$ is preserved the bosonic mass term $\omega \bar{\omega}$ implies the presence of the fermionic mass terms $\mu_{A} \bar{\mu}_{A}^{\prime}$ and $\mu_{A}^{\prime} \bar{\mu}_{A}$ to ensure invariance under the SUSY transformations (6).

Furthermore, one can also show that the ADHM-like couplings

$$
S_{E 2}^{A D H M}=\bar{\tau}_{\dot{\alpha}}^{A}\left(\bar{\omega}^{\dot{\alpha}} \mu_{A}+\bar{\mu}_{A} \omega^{\dot{\alpha}}\right)+i \vec{D} \cdot \omega \vec{\sigma} \bar{\omega}
$$

survives even for non-trivial intersections, where the latter interaction term describes the coupling of the bosonic modes to the auxiliary field $D^{\mu v}=\vec{D} \vec{\sigma}_{\mu v}$. Taking into account the supersymmetry transformation for $\bar{\tau}_{\dot{\alpha}}^{A}$

$$
\delta_{\bar{\xi}^{A}} \bar{\tau}=\bar{\xi}^{A} \vec{\sigma} \vec{D}
$$

it is easy to see that the interaction term (8) is supersymmetric if the E2-D6 system preserves $\bar{Q}_{\dot{\alpha}}^{A}$.

Let us now investigate whether or not a $U(1)$ instanton intersecting with a D-brane does contribute to the superpotential by performing the path integral over the instanton modes explicitly. Let us assume that the E2-D6 system preserves the supercharge $\bar{Q}_{\dot{\alpha}}^{0}$, which translates for our concrete setup (5) into the constraint on the angles $\sum_{I} \theta_{I}=0$. Moreover, we assume that the instantons are wrapping a rigid cycle thus apart from $\theta^{\alpha} \equiv \theta_{0}^{\alpha}$ and $\bar{\tau}_{\dot{\alpha}} \equiv \bar{\tau}_{\dot{\alpha}}^{0}$, the rest of the $\bar{\tau}^{A}, \theta_{A}$ modes are lifted by the holonomy of the background. 
The path integral takes the form

$$
\int d^{4} x d^{2} \theta d^{2} \bar{\tau} \prod_{A=0}^{3} d^{2} \omega d^{2} \bar{\omega} d \mu_{A}^{\prime} d \mu_{A} \prod_{B=0}^{2} d \bar{\mu}_{B}^{\prime} d \bar{\mu}_{B} d \bar{\mu}_{3} e^{-S_{E 2}^{m a s s}-S_{E 2}^{A D H M}} .
$$

After performing the integration over the $\bar{\tau}$ modes and $\mu^{0}, \bar{\mu}^{0}$ we are left with

$$
\int d^{4} x d^{2} \theta \prod_{A=1}^{3} d^{2} \omega d^{2} \bar{\omega} d \mu_{A}^{\prime} d \mu_{A} \prod_{B=1}^{2} d \bar{\mu}_{B}^{\prime} d \bar{\mu}_{B} d \bar{\mu}_{3} d \mu_{0}^{\prime} d \bar{\mu}_{0}^{\prime} e^{-S_{E 2}^{\text {mass }}}
$$

where we omit the term $\vec{D} \cdot \omega \vec{\sigma} \bar{\omega}$ which is irrelevant for the analysis. Next we use the mass terms for saturating the remaining fermionic charged instanton modes:

$$
\int d^{4} x d^{2} \theta \prod_{A=1}^{3} d^{2} \omega d^{2} \bar{\omega} d \bar{\mu}_{3} d \mu_{0}^{\prime} d \bar{\mu}_{0}^{\prime} e^{-\left(\bar{\mu}^{0} \mu_{0}^{\prime}+\mu^{0} \bar{\mu}_{0}^{\prime}\right)}
$$

Note that we cannot saturate the $\mu_{0}^{\prime}$ and $\bar{\mu}_{0}^{\prime}$ modes since we used already $\mu_{0}$ and $\bar{\mu}_{0}$ to saturate the universal $\bar{\tau}$ modes. Since there is no other way to soak up these modes the whole path integral vanishes. Thus we conclude a generic rigid $U(1)$ instanton does not contribute to the superpotential.

\section{EFFECTIVE FIELD THEORY ARGUMENT}

The previous discussion fits well into the general effective field theory analysis of [15, 32]. In particular, [32] argues that instantons which can become non-BPS (relative to the $\mathscr{N}=1$ background) as we move in closed string moduli space do not contribute to the superpotential, but contribute instead to Beasley-Witten higher F-terms [42, 43].

There are various important subtleties that one has to keep in mind when using this argument. First of all, when applying any misalignment argument one only needs to consider the subsector of the compactification putatively responsible for lifting the zero modes of the instanton. For instance, the example of a $U(1)$ instanton intersecting a brane discussed above will be generically part of some given compactification involving orientifold planes (away from the instanton), fluxes.6 and more background branes. In such a complete compactification, the physical closed string moduli space is very restricted (or possibly absent altogether, in the case where we stabilize all moduli), and typically one cannot misalign the instanton with the background while keeping supersymmetry. Nevertheless, those ingredients that have demonstrably no effect on $\bar{\tau}$ lifting can be ignored. Zero modes will be lifted in the complete compactification if and only if they are lifted in the absence of these elements of the compactification. The most important effect this has in practice is that we can generically ignore orientifold planes

\footnotetext{
6 As mentioned in the introduction, fluxes can change the analysis in important ways, and we do not address them in this section.
} 
away from the instanton. We only need to address the instanton and D-branes in a type II Calabi-Yau background.

Once we determine that the background orientifolds do not lift the zero modes of the instanton, the misalignment argument in [32] becomes very powerful: we can view our set of branes as a collection of $1 / 2$ BPS states of the $\mathscr{N}=2$ Calabi-Yau background, with its closed string moduli space intact. Moving in this closed string moduli space will change the argument of the central charge of the branes, differently for each homology class. Let us illustrate this in detail for type IIA compactifications. In this case the central charge of a brane (possibly instantonic) wrapping a 3-cycle $\Sigma$ in the internal Calabi-Yau is:

$$
Z=\int_{\Sigma} \Omega
$$

with $\Omega$ the complex structure of the Calabi-Yau. Type IIB at large volume work similarly, just replace $\Omega$ by $e^{-J+i(B-F)}$ in equation (13): 7

$$
Z=\int_{X} \operatorname{Tr} e^{-J+i(B-F)}
$$

with $X$ the cycle wrapped by the brane mirror to $\Sigma$, and $F$ the worldvolume flux on the mirror brane. In particular, note that the effect of a non-trivial worldvolume flux is to modify the effective homology class on which the brane is wrapped. At small volume the type IIB expression receives large $\alpha^{\prime}$ corrections, and it is more useful to map the problem to IIA using mirror symmetry.

Which $\mathscr{N}=1$ supersymmetry is preserved is determined by $\arg (Z)$, the phase of $Z$. This phase depends only on the homology class $[\alpha]$ of $\Sigma$, and our location in complex structure moduli space. Since in the absence of orientifolds we can move in arbitrary directions in complex structure moduli space freely. 8 any brane wrapping a homology cycle $[\beta] \neq[\alpha]$ can be misaligned from $\Sigma$. In particular, our instanton can be misaligned from any brane not in its homology class, and thus cannot have its $\bar{\tau}$ zero modes lifted by that brane (and thus, no superpotential can be generated). This leaves the cases where the brane wraps the same cycle as the instanton. The brane could be exactly on top of the instanton (the gauge and $U(1)$ cases described in the introduction) or it could be in a different cycle in the same homology class. We present an example of this last and somewhat more exotic case in the next section. An intriguing possibility, explored in [36], is to take the instanton and the brane to wrap cycles which are different in local homology, but are globally the same. We believe that in this case the effect of the background $B$-field should also be taken into account.

\footnotetext{
7 We have omitted some curvature terms that do not modify our discussion, see for example [44] for the complete expression.

${ }^{8}$ We have to take care of marginal stability walls along which the background "gauge" D-branes might decay, but generically they will not forbid all misalignment directions.
} 


\section{HOMOLOGICALLY GAUGE INSTANTONS}

Let us shortly review a different kind of instanton effect in type II which does contribute to the superpotential, introduced in [6].

Our starting point is $\mathscr{N}=4$ super-Yang mills. Instantons on top of the gauge brane have all their zero modes lifted due to interactions with the background gauge brane. In addition, they have the property that their moduli space consists of two components, a Higgs branch on which the instanton is dissolved into the gauge brane, and a Coulomb branch in which the instanton can be taken away from the brane. It is a simple calculation to show that even in the Coulomb branch the $\bar{\tau}$ modes are lifted by interactions. This can be shown in detail when the separation in the Coulomb branch is much smaller than the string length, in this case the relevant zero mode lifting comes from integrating out massive open strings between the instanton and the brane. Denoting the separation of the gauge brane and the instanton in the 6 internal directions as $\chi_{m}$ we obtain the following effective coupling in the instanton action lifting the $\bar{\tau}$ modes:

$$
e^{-S_{\text {inst }}\left(\chi_{m}\right)}=8 \pi^{2} g_{0}^{-3}(\bar{\tau})^{8}\left(32 t^{4}+144 t^{2}+64-\left(32 t^{5}+160 t^{3}+120 t\right) \sqrt{\pi} e^{t^{2}} \operatorname{erfc}(t)\right)
$$

where we have introduced the adimensional variable $t \equiv \chi^{2} / \sqrt{g_{0}}$ (with $g_{0}^{2}=$ $\left.4 \pi\left(4 \pi^{2} \alpha^{\prime}\right)^{-2} g_{s}\right)$, and $\operatorname{erfc}(t)$ denotes the complementary error function [45].

When the instanton and the brane are away from each other the dominant channel is massless closed string exchange at tree level between the instanton and the brane, with some insertions of $\bar{\tau}$ on the instanton boundary. The requisite diagrams have been computed in the context of M(atrix) theory [46] (for the coupling to the RR four-form) and early D(-1) instanton studies [47] (for the coupling to the metric), and they are nonvanishing:

$$
\begin{aligned}
& S_{i n s t} \sim \ldots+\partial_{m} \partial_{n} G_{\rho \sigma}\left(\bar{\Theta} \Gamma^{m \rho v} \Theta\right)\left(\bar{\Theta} \Gamma^{n \sigma v} \Theta\right) \\
& S_{\text {inst }} \sim \ldots+\partial_{m} \partial_{n} C_{\mu v \rho \sigma}^{(4)}\left(\bar{\Theta} \Gamma^{m \mu v} \Theta\right)\left(\bar{\Theta} \Gamma^{n \rho \sigma} \Theta\right)
\end{aligned}
$$

with $\Theta$ the spinor in the instanton worldvolume, which contains $\bar{\tau}$.

So far we have only dealt with $\mathscr{N}=4$, but the discussion will apply with little change to any instanton with a Coulomb branch. A particularly interesting class of examples comes from $\mathscr{N}=2 \mathrm{SYM}$. These theories can be engineered as D5 branes wrapping the blown up cycle of an $A_{1}$ singularity:

$$
x^{2}+y^{2}+w^{2}=0 .
$$

The interesting point is that this geometry can be easily fibered over the remaining one complex dimensional direction in order to obtain an $\mathscr{N}=1$ compactification with isolated spheres:

$$
x^{2}+y^{2}+w^{2}=f(z)^{2} .
$$

From the perspective of the worldvolume of the instanton, switching on $f(z)$ corresponds to lifting the Coulomb branch, leaving only a set of isolated points (the roots of $f(z)$ ). Since the original $\mathscr{N}=2$ configuration lifted the $\bar{\tau}$ modes everywhere in the Coulomb 
branch, we expect the $\bar{\tau}$ modes to be lifted on the deformed $\mathscr{N}=1$ geometry too, and thus to obtain contributions to the superpotential.

\section{LIFT OF $O(1)$ INSTANTONS TO F-THEORY}

In this section we will make some comments on how some known facts about D-brane instantons lift to F-theory. We will derive results which are already well understood from a CFT point of view in weakly coupled type II, but we do it from a purely target space point of view. In this way we will learn some lessons that carry over to situations where CFT techniques are not available.

In particular, we will discuss the F-theory lift of $O(1)$ instantons, and in particular how the orientifold removes the extra $\bar{\tau}$ fermionic zero modes. 9 The discussion is simplest in the flat space setting, so let us consider a F-theory compactification with base $\mathbb{R}^{6} \times T^{4}$. We will denote the coordinates $x^{0}, \ldots, x^{9}$ (with $x^{6}, \ldots, x^{9}$ parameterizing the $T^{4}$ torus). We want to lift the simplest $O(1)$ instanton, engineered as an instanton mapped to itself by the orientifold action. Let us choose the orientifold to be of the $\mathrm{O7}^{-}$type (we choose conventions such that a D7 on top of the orientifold has gauge group $O(1)$ ). This lifts in F-theory to a real codimension 2 degeneration of the fiber at $x^{8}=x^{9}=0$ (more precisely to a couple of degenerations, see below). The instanton with gauge group $O(1)$ will then be an euclidean D3 brane 10 wrapping the $T^{4}$.

We would like to argue from a target space viewpoint that the orientifold projects out the $\bar{\tau}$ modes present in the absence of the $\mathrm{O7}^{-}$. Consider the monodromy around the $O 7^{-}$, it is given by the following $\operatorname{SL}(2, \mathbb{Z})$ element, up to conjugation:

$$
M_{O}=\left(\begin{array}{cc}
-1 & 4 \\
0 & -1
\end{array}\right)
$$

where the 4 encodes the RR charge of the $07^{-}$. The diagonal -1 part is the F-theory lift of the $(-1)^{F_{L}} \cdot \Omega$ orientifold action in type IIB [49], and will thus be responsible for projecting out the $\bar{\tau}$ modes from the F-theory perspective, as we show below.

The monodromy (19) encodes the action of S-duality in the IIB picture, and induces a S-duality action on the euclidean D3 worldvolume. Let us describe this worldvolume theory in some detail. Before introducing the orientifold, we would have euclidean $\mathscr{N}=4 U(1)$ Yang-Mills theory living in a $T^{4}$. From the point of view of this theory, introducing the orientifold amounts to introducing a surface defect operator with the property that as we go around it the fields in the $\mathscr{N}=4$ theory enjoy a monodromy

\footnotetext{
9 As we will see below, the configuration that we study becomes a smooth F-theory model after quantum corrections, so Witten's arithmetic genus criterion [48] can be applied to the generic $O(1)$ instanton. We will choose instead to work more microscopically. While the analysis is more involved in this way, it will illuminate some aspects of the instanton worldvolume theory which are not easy to see by looking just at the symmetries.

${ }^{10}$ We choose to work in a language close to type IIB. In the M-theory description we would have a M5 wrapping $T^{4} \times T^{2}$. After compactification of the 6 dimensional theory on the worldvolume of the M5 down to 4 dimensions (by compactification on the $T^{2}$ fiber), the discussion below would also apply to the M-theory setting.
} 
given by $M_{O}$. Furthermore, the defect will break supersymmetry down to $\mathscr{N}=2$ (as we can easily see from the stringy description), and it makes the theory non-conformal. We will discuss in detail the origin and consequences of this non-conformality below.

It is hard to give a detailed description of the euclidean D3 brane worldvolume theory with surface defects, however these simple properties described above will suffice to analyze the effect of the orientifold on zero modes. First of all, from [50] for example, we learn that $M_{O}$ acts on the supersymmetries $Q_{\alpha}, \tilde{Q}_{\dot{\alpha}}$ of $\mathscr{N}=4 \mathrm{SYM}$ in a chiral way (here, and in the following, we will keep the $S U(4)_{R}$ spinor index on the $\mathscr{N}=4$ spinors implicit). Writing the general monodromy matrix as

$$
M=\left(\begin{array}{ll}
a & b \\
c & d
\end{array}\right)
$$

the action of $M$ on supersymmetry charges is of the form

$$
Q_{\alpha} \rightarrow e^{i \varphi(M)} Q_{\alpha}, \quad \tilde{Q}_{\dot{\alpha}} \rightarrow e^{-i \varphi(M)} \tilde{Q}_{\dot{\alpha}}
$$

with 11

$$
e^{i \varphi(M)}=\left(\frac{|c \tau+d|}{c \tau+d}\right)^{1 / 2} .
$$

In our case, this gives

$$
Q_{\alpha} \rightarrow \sqrt{-1} Q_{\alpha}, \quad \tilde{Q}_{\dot{\alpha}} \rightarrow-\sqrt{-1} \tilde{Q}_{\dot{\alpha}}
$$

Notice that this rotation is chiral, acting differently on right handed and left handed fermionic supercharges. In the case of $\mathscr{N}=4$ eq. (23) is actually a harmless phase redefinition, since it is contained in the center of the (anomaly-free) $S U(4)$ R-symmetry group.

We have given the action of the orientifold S-duality on the supersymmetries. The $\bar{\tau}, \theta$ modes that we are interested in are in this language the gauginos of the $\mathscr{N}=4$ theory. They are obtained by acting with the supersymmetric transformations on the gauge bosons, and thus transform just as the supercharges:

$$
\bar{\tau} \rightarrow \sqrt{-1} \bar{\tau}, \quad \theta \rightarrow-\sqrt{-1} \theta .
$$

We will now argue that the action (23) is actually anomalous in our $\mathscr{N}=2$ setting, and thus physical (in the sense that the associated monodromy projects out states). The key point is that (24) is no longer anomaly-free after the insertion of defects. Naively this would be easy to argue, since the rotation that undoes (24) is part of the $U(1)_{R}$ classical symmetry of $\mathscr{N}=2$ gauge theories, which is in the same multiplet as the stress-energy tensor, and thus is anomalous if the theory is non-conformal (which our theory is).

However, the analysis is more involved. The gauge instantons that break $U(1)_{R}$ leave a discrete subgroup invariant. In the case of ordinary $S U(N) \mathscr{N}=2$ with flavors the

\footnotetext{
11 The choice of notation is standard but somewhat unfortunate. $\bar{\tau}$ refers to the fermionic zero modes of the instanton, while $\tau$ denotes the complexified gauge coupling of $\mathscr{N}=4$ super-Yang-Mills.
} 
anomaly-free subgroup always contains the $\mathbb{Z}_{4}$ action (24). So the last step that we need to show is that in the presence of the orientifold defect, this universal $\mathbb{Z}_{4}$ subgroup is actually partially broken to $\mathbb{Z}_{2}$ by anomalies $\left(\mathbb{Z}_{2}\right.$ always survives, as it corresponds to multiplying all fermions by -1 ).

Let us present some evidence that this is actually the case. First of all, let us use the fact that the $\mathrm{O7}^{-}$plane splits into two separated degenerations once we lift it to F-theory [49, 51] (we will refer to these two degenerations as the elementary defects). In order to be explicit, let us take the conventions of [52]. In these conventions, the $\mathrm{OT}^{-}$plane splits into a $(1,-1)$ brane and a $(1,1)$ brane. Let us denote them the $B$ and $C$ branes, respectively. See figure 1 for an illustration of the system after the orientifold splits.

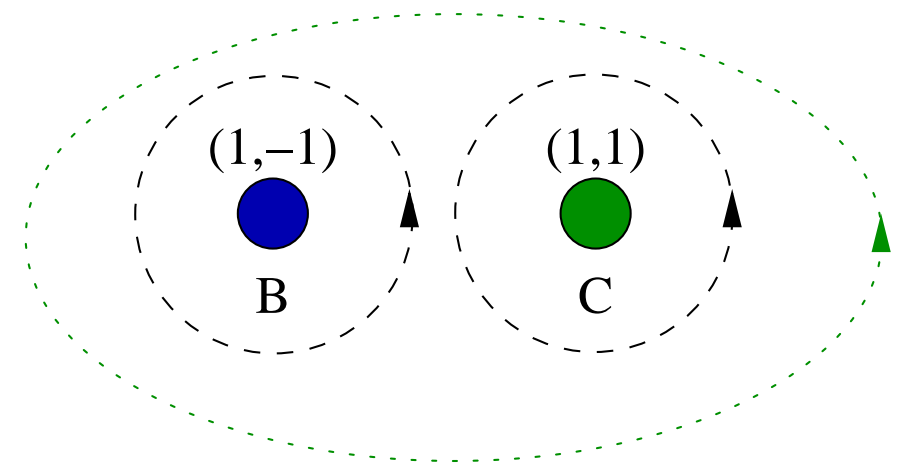

FIGURE 1. Schematic representation of the Euclidean D3-brane worldvolume theory after the orientifold splits, close to the orientifold locus. The two dots denote the location of the $B$ and $C$ defects. We have omitted the directions common to the $\mathrm{O}^{-}$and the Euclidean D3. The dotted green line denotes the contour along which we compute the monodromy. It factorizes into the two smaller dashed contours around each elementary degeneration.

Recalling that the monodromy for a generic $(p, q)$ brane is given (in a convenient $S L(2, \mathbb{Z})$ frame) by:

$$
M_{(p, q)}=\left(\begin{array}{cc}
1-p q & p^{2} \\
-q^{2} & 1+p q
\end{array}\right)
$$

we have the following monodromies for the $B$ and $C$ branes:

$$
B=M_{(1,-1)}=\left(\begin{array}{cc}
2 & 1 \\
-1 & 0
\end{array}\right) \quad C=M_{(1,1)}=\left(\begin{array}{cc}
0 & 1 \\
-1 & 2
\end{array}\right)
$$

where we have abused notation slightly, and denoted by $B$ the monodromy matrix for $B$ branes, and similarly for $C$ branes. It is easy to check that indeed $B C=M_{O}$, so the orientifold decomposes into these two degenerations. We can now decompose the $U(1)_{R}$ rotation of the supersymmetries due to the $S L(2, \mathbb{Z})$ transformation into a couple of consecutive rotations, as we go around each degeneration separately:

$$
e^{i \varphi\left(O 7^{-}\right)}=\sqrt{-1}=e^{i \varphi\left(M_{B}\right)} e^{i \varphi\left(M_{C}\right)}
$$


where

$$
\begin{aligned}
e^{i \varphi\left(M_{C}\right)} & =\sqrt{\frac{|-\tau+2|}{-\tau+2}} \\
e^{i \varphi\left(M_{B}\right)} & =\sqrt{\frac{\left|\tau_{C}\right|}{-\tau_{C}}} .
\end{aligned}
$$

We have introduced $\tau_{C}=1 /(-\tau+2)$, the value of the coupling after going around $C$. From these expressions we see that we can undo the $U(1)_{R}$ rotation in two steps: first undo the rotation due to $B$, and then the rotation due to $C$ (or vice versa). The overall rotation will be anomalous if the addition of the induced shifts to the $\mathscr{N}=2 \theta$ angle is not a multiple of $2 \pi 12$

From the expressions above, for generic coupling, we see that it is enough that the shift of the $\mathscr{N}=2 \theta$ angle is not of the form $4 \times \varphi$ (here $\varphi$ denotes the $U(1)_{R}$ rotation angle), as it would be for usual $\mathscr{N}=2$ theory with flavors, but $2 \times \varphi$. This is actually a plausible result, for the following reason. The factor of 4 comes from the number of zero modes in the one instanton background in which we compute the anomaly. The background in which we want to compute our anomaly is not a one-instanton background, but rather a one-vortex background. There is a deep relation between vortices and instantons (see for example [53]), and in particular the dimension of the $k$-instanton moduli space is $4 k N_{c}$, while the dimension of the $k$-vortex moduli space is $2 k N_{c}$ (in our case we have $N_{c}=k=1$ ). In situations with enough supersymmetry, the bosonic dimension of the moduli space agrees with the fermionic dimension of the moduli space, and thus we expect that the number of fermionic zero modes of a vortex is exactly half the number of fermionic zero modes of an instanton, leading to half a contribution to the anomaly.

Based on this evidence (and from our knowledge of the microscopic picture) this leads us to argue that the correct shift of the axion in the vortex background is indeed $2 \varphi$, and thus the action of $S L(2, \mathbb{Z})$ cannot be undone by an anomaly-free $U(1)_{R}$ rotation.

Equipped with these arguments, we see that upon circling once around the defect, the $\mathscr{N}=4$ field theory gets mapped to itself, except for the chiral rotation of the gauginos. We can multiply all fermions by $\sqrt{-1}$ (a harmless phase redefinition 13 ), and we obtain the following monodromy action:

$$
\theta \rightarrow \theta, \quad \bar{\tau} \rightarrow-\bar{\tau}
$$

Notice that we are mapping the gauge coupling to itself: $\tau \rightarrow \tau-4$ (the integer shift in the theta angle has no effect on the theory), and in particular far away from the orientifold

\footnotetext{
12 Here we are assuming that the anomaly we get when undoing the $U(1)_{R}$ rotation due to each elementary defect comes only from zero modes localized at that defect. A possible justification for this assumption comes from the fact that the two defects are mutually non-local, and only the zero modes corresponding to one of them (in a particular frame where it is of type $(1,0)$ ) should be considered dynamical. This is consistent with the fact that in the F-theory lift there is a single complex structure modulus controlling the position of both elementary degenerations, once we fix the string coupling [49].

13 At this point we could have also chosen $-\sqrt{-1}$. The sign is determined by imposing that the resulting projection coming from (30) is the one compatible with the supersymmetries preserved by the instanton$O 7^{-}$system.
} 
we are mapping weak coupling to weak coupling, so we can identify degrees of freedom straightforwardly. The minus sign in front of $\bar{\tau}$ then tells us that this mode is unphysical, and projected out by the orientifold.

Note also that the crucial point was the $\sqrt{-1}$ component of the orientifold monodromy action (23) on the fermions, which does not act on the gauge coupling. This $\sqrt{-1}$ factor is absent in the case of pure D7 branes, where we have $e^{i \varphi(M)}= \pm \sqrt{1}$, giving a non-chiral (and thus anomaly-free) phase rotation. In fact this is precisely the anomaly-free $\mathbb{Z}_{2}$ subgroup that was preserved by the orientifold.

\section{ACKNOWLEDGMENTS}

We would like to acknowledge interesting discussions with Ralph Blumenhagen, Jim Halverson, Liam McAllister, Tony Pantev, Mike Schulz, Angel Uranga, and Timo Weigand. We would also like to thank Jonathan Heckman, Joseph Marsano, Natalia Saulina, Sakura Schäfer-Nameki and Cumrun Vafa for extensive discussions after [6] appeared. I.G.E. thanks Nao Hasegawa for kind support and constant encouragement. This work is supported by DOE grant DE-FG05-95ER40893-A020, NSF RTG grant DMS-0636606 and Fay R. and Eugene L. Langberg Chair.

\section{REFERENCES}

1. K. Becker, M. Becker, and A. Strominger, Nucl. Phys. B456, 130-152 (1995), hep-th/9507158.

2. R. Blumenhagen, M. Cvetič, and T. Weigand, Nucl. Phys. B771, 113-142 (2007), hep-th/0609191.

3. L. E. Ibáñez, and A. M. Uranga, JHEP 03, 052 (2007), hep-th/ 0609213.

4. B. Florea, S. Kachru, J. McGreevy, and N. Saulina, JHEP 05, 024 (2007), hep-th/ 0610003.

5. R. Blumenhagen, M. Cvetič, S. Kachru, and T. Weigand (2009), 0902.3251.

6. M. Cvetič, I. García-Etxebarria, and R. Richter (2009), 0905.1694.

7. E. Witten, Nucl. Phys. B460, 541-559 (1996), hep-th/9511030.

8. M. R. Douglas, J. Geom. Phys. 28, 255-262 (1998), hep-th/9604198.

9. M. Billò, et al., JHEP 02, 045 (2003), hep-th/0211250.

10. N. Akerblom, R. Blumenhagen, D. Lüst, E. Plauschinn, and M. Schmidt-Sommerfeld, JHEP 04, 076 (2007), hep-th/0612132.

11. M. Bianchi, and E. Kiritsis, Nucl. Phys. B782, 26-50 (2007), hep-th/ 0702015.

12. R. Argurio, M. Bertolini, G. Ferretti, A. Lerda, and C. Petersson, JHEP 06, 067 (2007), 0704.0262

13. M. Bianchi, F. Fucito, and J. F. Morales, JHEP 07, 038 (2007), 0704.0784.

14. M. Aganagic, C. Beem, and S. Kachru, Nucl. Phys. B796, 1-24 (2008), 0709.4277.

15. I. García-Etxebarria, and A. M. Uranga, JHEP 01, 033 (2008), 0711.1430.

16. C. Petersson, JHEP 05, 078 (2008), 0711 . 1837 .

17. I. García-Etxebarria, JHEP 07, 017 (2009), 0810.1482 .

18. G. Ferretti, and C. Petersson, JHEP 03, 040 (2009), 0901.1182

19. R. Argurio, M. Bertolini, S. Franco, and S. Kachru, JHEP 06, 017 (2007), hep-th/ 0703236.

20. L. E. Ibáñez, A. N. Schellekens, and A. M. Uranga, JHEP 06, 011 (2007), 0704.1079.

21. D. Marolf, L. Martucci, and P. J. Silva, JHEP 04, 051 (2003), hep-th/0303209

22. D. Marolf, L. Martucci, and P. J. Silva, JHEP 07, 019 (2003), hep-th/ 0306066

23. R. Kallosh, A.-K. Kashani-Poor, and A. Tomasiello, JHEP 06, 069 (2005), hep-th/ 0503138.

24. L. Martucci, J. Rosseel, D. Van den Bleeken, and A. Van Proeyen, Class. Quant. Grav. 22, 2745-2764 (2005), hep-th/0504041.

25. E. Bergshoeff, R. Kallosh, A.-K. Kashani-Poor, D. Sorokin, and A. Tomasiello, JHEP 10, 102 (2005), hep-th/0507069. 
26. J. Park (2005), hep-th/0507091

27. I. Bandos, and J. A. de Azcarraga, JHEP 09, 064 (2005), hep-th/0507197

28. D. Lüst, S. Reffert, W. Schulgin, and P. K. Tripathy, JHEP 08, 071 (2006), hep-th/0509082.

29. D. Tsimpis, JHEP 03, 099 (2007), hep-th/ 0701287.

30. W. Schulgin, Nucl. Phys. Proc. Suppl. 171, 316-318 (2007).

31. R. Blumenhagen, M. Cvetič, R. Richter, and T. Weigand, JHEP 10, 098 (2007), 0708.0403 ,

32. I. García-Etxebarria, F. Marchesano, and A. M. Uranga, JHEP 07, 028 (2008), 0805.0713.

33. M. Billo', et al., JHEP 12, 102 (2008),0807.4098.

34. M. Billo', et al., JHEP 10, 112 (2008), 0807.1666 .

35. A. M. Uranga, JHEP 01, 048 (2009), 0808.2918.

36. J. J. Heckman, J. Marsano, N. Saulina, S. Schäfer-Nameki, and C. Vafa (2008), 0808.1286.

37. J. Marsano, N. Saulina, and S. Schäfer-Nameki (2008), 0808.2450

38. T. Banks, L. J. Dixon, D. Friedan, and E. J. Martinec, Nucl. Phys. B299, 613-626 (1988).

39. T. Banks, and L. J. Dixon, Nucl. Phys. B307, 93-108 (1988).

40. M. Cvetič, R. Richter, and T. Weigand, Phys. Rev. D76, 086002 (2007), hep-th/0703028

41. M. B. Green, and M. Gutperle, JHEP 02, 014 (2000), hep-th/ 0002011

42. C. Beasley, and E. Witten, JHEP 01, 056 (2005), hep-th/ 0409149.

43. C. Beasley, and E. Witten, JHEP 02, 060 (2006), hep-th/ 0512039

44. P. S. Aspinwall (2004), hep-th/0403166.

45. M. Abramowitz, and I. A. Stegun, Handbook of mathematical functions with formulas, graphs, and mathematical tables, http://www.math.sfu.ca/ cbm/aands/(1964).

46. K. Millar, W. Taylor, and M. Van Raamsdonk (2000), hep-th/ 0007157.

47. M. B. Green, and M. Gutperle, Nucl. Phys. B498, 195-227 (1997), hep-th/9701093.

48. E. Witten, Nucl. Phys. B474, 343-360 (1996), hep-th/9604030

49. A. Sen, Nucl. Phys. B475, 562-578 (1996), hep-th/9605150.

50. A. Kapustin, and E. Witten (2006), hep-th/0604151.

51. N. Seiberg, and E. Witten, Nucl. Phys. B426, 19-52 (1994), hep-th/9407087

52. M. R. Gaberdiel, T. Hauer, and B. Zwiebach, Nucl. Phys. B525, 117-145 (1998), hep-th/9801205.

53. D. Tong (2005), hep-th/0509216 